\title{
Correlation Averaging of Single-Atomic-Column STEM-EDX Images for Sub-Atomic Information
}

\author{
Jong Seok Jeong ${ }^{1}$ and K. Andre Mkhoyan ${ }^{1}$ \\ 1. Department of Chemical Engineering and Materials Science, University of Minnesota, \\ Minneapolis, MN 55455, United States.
}

With recent advances in analytical scanning transmission electron microscopy (STEM), including use of aberration correction of the lenses and high-efficiency electron energy-loss (EEL) and energy dispersive X-ray (EDX) spectrometers, it becomes routine practice to obtain atomic-resolution compositional maps [1-3]. This opens possibilities for extracting sub-atomic information from such maps. Efforts to retrieve sub-atomic information from STEM-EELS spectrum images by deconvolving channeled STEM probes from the spectrum images have been made [4-6]. However, acquiring experimental low-noise, atomic-resolution maps for such analyses has been challenging, and the outcomes have been suitable only for basic qualitative interpretation. A key challenge for achieving sub-atomic information is the improvement of signal-to-noise ratio (SNR) of compositional maps. Here, we report a simple and reliable solution for achieving high-SNR EDX spectrum images for individual atomic columns. The method is based on standard cross-correlation, aided by averaging of single atomic column EDX maps with modifications in the reference image. Step-by-step procedures and the reliability, stability, and limitation of the method, are presented.

An aberration-corrected FEI Titan G2 60-300 STEM, operated at $300 \mathrm{keV}$, was used in this study. STEM-EDX maps were obtained using the FEI Super-X system, a quad-silicon-drift windowless in-polepiece EDX detector. With the beam current of $150 \mathrm{pA}$ and dwell time of 3 $\mu$ s/pixel, $256 \times 256$ pixel $^{2}$ EDX maps were acquired from areas $32 \times 32 \AA^{2}$ of a $\mathrm{SrTiO}_{3}$ crystal. Frame by frame drift correction using the Bruker Esprit 1.9 software was performed.

The method in this work uses a self-consistently determined reference map to cross-correlate many individual noisy maps that can be cut out from a few experimental STEM-EDX maps with relatively small scan area. The size of the reference image plays a critical role in the crosscorrelation process. To determine unbiasedly the proper size of the gaussian reference image, two different approaches are employed and they are in excellent agreement (Fig. 1).

Figure 2 shows the evaluated peak SNR (PSNR) as a function of the number of images averaged. Interestingly, it is noted that a small difference in the SNR of raw maps can result in a considerable difference in the number of maps required to reach the same level of PSNR, as it is the case for $\mathrm{Sr} L$ and $\mathrm{Ti} K \alpha$ maps. One of the examples for SNR-improved experimental EDX maps is shown in Fig. 3. Nine sets of EDX maps were averaged to obtain SNR-improved maps (Fig. 3a), and then they are further averaged ('Final' in Fig. 3a). It is clearly seen that the SNR was dramatically improved in the final averaged maps so that small differences in sizes between maps from $K$ and $L$ shells of each atomic column can be resolved (Fig. 3b). These high-SNR maps can be further analyzed for sub-unit cell information. For example if effects of channeling probe are properly removed, the excitation potentials can be extracted [7]. 
References:

[1] DA Muller et al, Science 319 (2008), p. 1073

[2] AJ D'Alfonso et al, Phys. Rev. B 81 (2010), p. 100101

[3] K Suenaga et al, Nat. Photon 6 (2012), p. 545

[4] MJ Neish et al, Phys. Rev. B 88 (2013), p. 115120

[5] G Kothleitner et al, Phys. Rev. Lett. 112 (2014), p. 085501

[6] NR Lugg et al, Microsc. Microanal. 20 (2014), p. 1078

[7] This work was supported by NSF MRSEC under award DMR-1420013. STEM analysis was carried out in the Characterization Facility of the University of Minnesota, which receives partial support from the NSF through the MRSEC program.

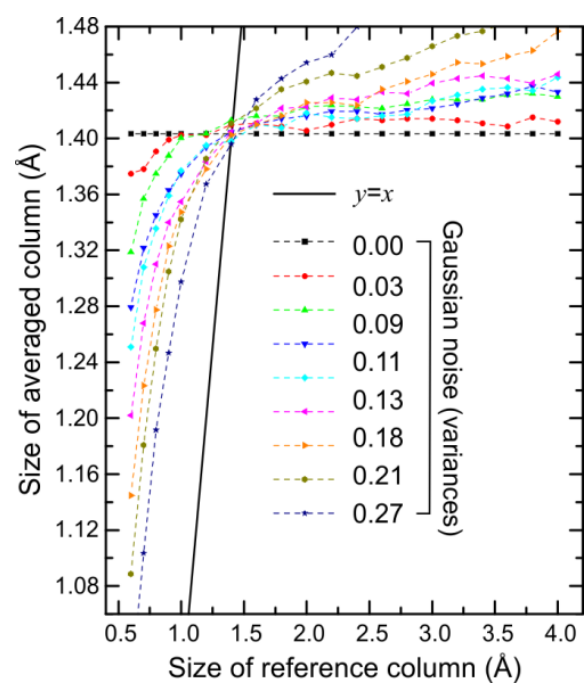

Figure 1. The size of averaged columns as a function of the size of reference columns used. The cross-over indicates the selfconsistent size determination point.

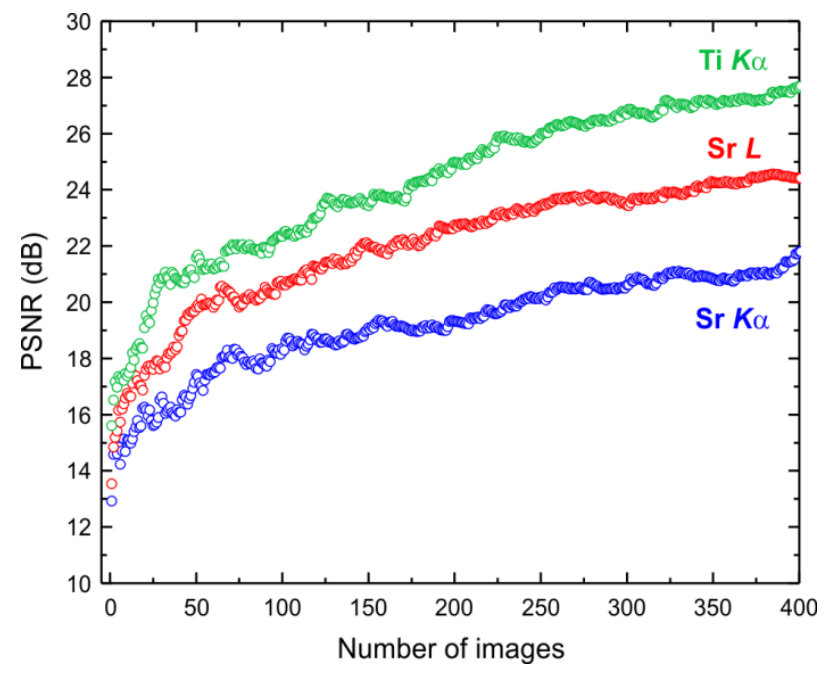

Figure 2. PSNR as a function of the number of single-atomic-column EDX images averaged.
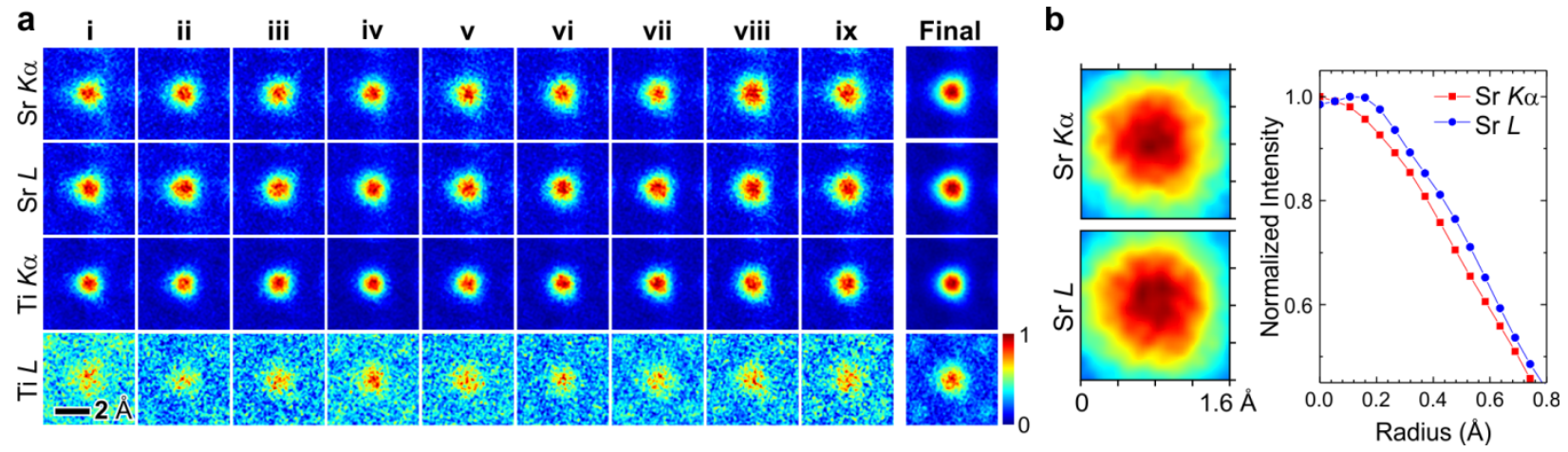

Figure 3. (a) SNR-improved single-atomic-column $\mathrm{Sr} K \alpha / L$ and $\mathrm{Ti} K \alpha / L$ EDX maps obtained from nine different runs of mapping. Final SNR-improved data from each peak of $\mathrm{Sr}$ and $\mathrm{Ti}$ are presented. (b) Magnified SNR-improved EDX maps from $\mathrm{Sr} K \alpha$ and $L$ peaks obtained from the 'Final' in (a) alongside their corresponding azimuthally averaged radial profile. 